\title{
L'individu comme problème phénoménologique chez Hannah Arendt et Michel Henry
}

Jan Černý

Journal of French and Francophone Philosophy - Revue de la philosophie

française et de langue française, Vol XX, No 2 (2012) pp 19-41

\author{
Vol XX, No 2 (2012) \\ ISSN 1936-6280 (print) \\ ISSN 2155-1162 (online) \\ DOI 10.5195/jffp.2012.536 \\ www.jffp.org
}

\section{(oc) EY-NC-ND}

This work is licensed under a Creative Commons Attribution-Noncommercial-No Derivative Works 3.0 United States License.

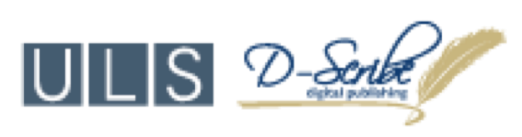

This journal is operated by the University Library System of the University of Pittsburgh as part of its D-Scribe Digital Publishing Program, and is co-sponsored by the University of Pittsburgh Press 


\title{
L'individu comme problème phénoménologique chez Hannah Arendt et Michel Henry
}

\author{
Jan Černý
}

Charles University, Prague

Le trait caractéristique unissant la philosophie politique de Hannah Arendt et la "phénoménologie matérielle"1 de Michel Henry est la défense de l'unicité de l'individu, inconvertible en un réseau de déterminations sociales, économiques, discursives ou autres. Contrairement à bien d'autres philosophes de leur temps, ni Arendt ni Henry n'ont perdu la foi en le "je peux" fondamental dont est doté chaque sujet naissant dans l'action politique ou l'immanence de la vie.

Bien que les deux penseurs s'intéressent à la base à des problématiques philosophiques différentes et que la subjectivité intervienne chez eux dans d'autres contextes thématiques, leurs approches se croisent dans deux moments essentiels: (i) ils relient la naissance du sujet individuel humain à un mode d'apparaître spécifique, dont l'essence est la manifestation de l'individu elle-même; (ii) ils appréhendent l'évolution culturelle moderne comme une menace substantielle des conditions de l'apparaître que l'individu crée de manière irremplaçable et insubstituable et par lesquelles il se manifeste.

Le diagnostic critique de la culture moderne se fait, chez les deux auteurs, à partir d'un présupposé ontophénoménologique similaire: l'Être est un avec l'apparaître ${ }^{2}$ et la subjectivité humaine est pensée comme une région unique de l'être, plus ou moins déconnectée des autres régions, ces dernières étant reliées à d'autres conditions d'apparaître. Ils comprennent la culture moderne comme un oubli de cette région unique ainsi que des conditions d'apparence propres à l'individu.

Cet article ne tente pas une comparaison entre deux projets philosophiques différents, c'est-à-dire entre la philosophie politique de Hannah Arendt et la phénoménologie matérielle de Michel Henry; son but

Journal of French and Francophone Philosophy | Revue de la philosophie française et de langue française Vol XX, No 2 (2012) | www.jffp.org | DOI 10.5195/jffp.2012.536 
est de montrer que Henry et Arendt ont, indépendamment l'un de l'autre, remis en question la conviction traditionnelle du primat ontologique d'un fondement inapparent, qui dirige les conditions sous lesquelles se donne le phénomène, et ils ont situé la plénitude de l'être dans l'autodonation spontanée de ce phénomène qu'est selon eux l'individu lui-même. Ce faisant, ils ont opposé la puissance positive de l'apparaître de l'individu aux forces aliénatrices libérées par le développement de la technoscience moderne.

Dans cette étude je voudrais, dans un premier temps, apporter des preuves à la possibilité d'interpréter la pensée politique de Arendt comme un projet phénoménologique original dont le but est d'élever l'apparence de la personne au rang de mode unique de l'apparaître. Puis je présenterai brièvement la phénoménologie matérielle de M. Henry dans laquelle le Soi individuel joue un rôle tout aussi central, puisqu'il est la condition de l'apparence de la vie et le fondement de tout apparaître. En conclusion, j'esquisserai les conséquences d'une telle position privilégiée du sujet individuel pour la conception théorique de la réalité effective de l'apparaître, de même que pour certains problèmes pratiques de l'action de l'homme dans le monde.

\section{Hannah Arendt: le ménage et le domaine public}

La philosophie politique de Hannah $\operatorname{Arendt}^{3}$ est bâtie sur la conviction, qui a d'après elle son origine dans la praxis politique grecque, c'est-à-dire sur la conviction d'une séparation stricte entre le domaine public (der öffentliche Raum), où se produit la vie politique, et la sphère privée du ménage (der Haushalt) où l'on assure la simple conservation de la vie. Selon son interprétation, une hiérarchie claire régnait entre les deux sphères: alors que dans la sphère du ménage l'homme était soumis aux "contraintes vitales" ${ }^{4}$ (aux préoccupations pour la conservation de l'individu et du genre, et donc au travail et à la reproduction), le domaine public de la polis antique, lui, était le royaume de la liberté où l'homme s'affranchit de la nécessité dans laquelle l'enferme le souci de la conservation de la vie. Bien que la vie du ménage soit de par sa nature une vie au sein d'une communauté de genre, les Grecs la définissaient comme un intérêt pour ce qui est idion, purement propre à l'homme, privé. Tout souci de la propriété et de la richesse $^{5}$ en fait donc également partie. Dans la vie politique dans le domaine public l'homme libre s'occupait au contraire de ce qui était "commun" (koinon), à la chose commune de la cité. Alors que le règne de la nécessité de la conservation de la vie dans la sphère privée crée des rapports de contrainte, des rapports maître - esclave, homme - femme, ${ }^{6}$ dans le domaine public le citoyen libre rencontre (et agit avec) des citoyens libres qui lui sont égaux et qui ont laissé la nécessité vitale et les rapports qu'elle établit derrière le seuil de leurs ménages.

Journal of French and Francophone Philosophy | Revue de la philosophie française et de langue française Vol XX, No 2 (2012) | http://www.jffp.org | DOI 10.5195/jffp.2012.536 
Arendt caractérise la fondation différente des deux sphères et la nécessité de leur séparation stricte à l'aide de catégories phénoménales - le ménage est la sphère de l'obscurité et de la touffeur, alors que le domaine public de la polis est la sphère de la lumière et de la manifestation. Arendt trouve une double motivation à "l'obscurité" du ménage: le ménage est d'abord un lieu caché où a lieu l'acte sacré de la naissance et de la mort; car les hommes mortels sont ceux "qui, de même que toute créature, sortent des ténèbres et retournent aux ténèbres du monde souterrain. ${ }^{77}$ Le mystère $d u$ début et de la fin de la vie humaine doit être mis à l'abri de la lumière du domaine public. La seconde motivation de la touffeur de la sphère du ménage est la pudeur - se réfugie dans le ménage ce qui est honteux, les besoins corporels et leur satisfaction, et puis généralement ce qui est nécessaire et fugace, le souci de la vie qui se joue dans le cercle de la faim et du rassasiement et dans le cercle du travail, de la fatigue et du repos. C'est seulement dans le domaine public que l'homme perce la cyclicité dénuée de sens de sa vie biologique en créant (pour être plus précis, en co-créant avec les autres) l'histoire pleine de sens de sa vie et l'Histoire de sa cité.

Le domaine public est inversement la sphère de la lumière et de la manifestation, et constitue en ce sens la contradiction exacte du ménage. Et cette contradiction signifie pour Arendt surtout un degré d'être différent: la réalité des choses qui ont été vues et entendues par le "public" est incomparable avec ce qui est purement privé. La réalité de quelque chose au sens fort est garantie par le paraître de cette chose pour les autres. Dans un autre passage Arendt formule explicitement le principe de l'identité de l'être et du paraître: "Dans ce monde où nous entrons, apparus de nulle part, et dont nous disparaissons en direction de nulle part, Etre et Paraître coïncident. ${ }^{8}$ Le deuxième trait fondamental du domaine public, à savoir le fait que la sphère publique constitue le monde en tant que ce qui nous est commun, est lui aussi présenté d'un point de vue phénoménal - est commun précisément ce qui se manifeste à tous ensemble. Cette communauté du monde commun fonde un moment crucial pour Arendt, le moment de la pluralité: la pluralité de ceux qui voient et de là la pluralité des perspectives depuis lesquelles telle chose ou telle personne sont vues. ${ }^{9}$ Le monde commun naît comme un réseau de perspectives diverses depuis lesquelles est appréhendée telle chose.

\section{L'action en tant que révélation de l'agent}

Sur le fond de ce moment de l'automanifestation réciproque des hommes dans le domaine public Arendt développe sa remarquable anthropologie, au moyen de laquelle elle tente de dépasser la tradition de la philosophie occidentale qui "n'est capable de parler de l'homme qu'au singulier et s'occupe $\mathrm{du}$ fait de la pluralité uniquement de manière marginale." ${ }^{10}$ Ce n'est que dans le domaine public que nous acquérons notre

Journal of French and Francophone Philosophy | Revue de la philosophie française et de langue française Vol XX, No 2 (2012) | http://www.jffp.org | DOI 10.5195/jffp.2012.536 
22 L’individu comme problème phénoménologique chez Hannah Arendt et Michel Henry

identité la plus propre: c'est là qu'a lieu la "révélation de l'agent" (die Enthüllung der Person), la réponse à la question implicite du public “Qui estu?" Cela requiert un développement plus détaillé. Le domaine public est pour Arendt l'espace d'une activité qu'elle nomme l'action (das Handeln). La définition de cette dernière est une fois de plus dérivée de la pratique politique grecque et elle comprend deux activités qui portent une vie politique libérée des liens de la nécessité - l'action (praxis, Handeln) et la parole (lexis, Sprechen). En Grèce antique, l'action et la parole étaient considérées comme les deux plus grandes facultés humaines dès l'époque de Homère, puisqu'elles permettaient aux hommes de se faire remarquer et accéder à la gloire, et de s'assurer ainsi l'immortalité dans la mémoire de la cité, ce qui représentait le vrai but de la vie politique et de la vie d'un citoyen libre de manière générale. L'action et la parole sont étroitement liées: la parole en tant que persuasion des autres est un mode selon lequel se produit l'action politique - seule une telle action s'abstient d'utiliser des moyens violents et demeure effectivement politique. ${ }^{11}$

L'action et la parole ont, ce faisant, un certain excès sur elles-mêmes: l'action est davantage que l'atteinte d'une fin (s'il en était ainsi elle pourrait aisément se transformer en violence muette), la parole est davantage que la transmission d'une information (ainsi elle pourrait aisément être remplacée par le langage des signes). Si Arendt affirme que "la parole et l'action [...] sont les modes sous lesquels les êtres humains apparaissent les uns aux autres", ${ }^{12}$ nous pouvons estimer que c'est peut-être justement cet excès (de signification, d'apparence) qui individualise chaque personne, fait qu'elle est inaccessible et que précisément cette individualisation excessive, débordante des personnes constitue l'essence et la fin de l'humain. C'est le fait du domaine public et de la pluralité des hommes en lui qui est ici constitutif - la multitude des personnes qui prennent part (qui regardent et agissent) constitue la clarté de la vie publique au sein de laquelle peut apparaître un individu concret, inaccessible. La révélation de l'agent n'est, ce faisant, pas le dessein propre de l'action, il se produit pour ainsi dire involontairement, dans l'engouement de sa propre action et uniquement pour les autres, comme il s'ensuit de sa détermination phénoménale.

Cette conception de la constitution de la personne à travers son apparence dans l'espace public prend d'après moi son origine dans une autre dichotomie séparant deux sphères de manière stricte, à savoir dans l'opposition traditionnelle entre l'être et le paraître. Dans son anthropologie Arendt renverse la hiérarchie entre l'être "véritable" et le "seul" paraître. Elle défend explicitement ce procédé dans son livre The Life of the Mind: elle qualifie d'abord la conviction des métaphysiques traditionnelles sur "la suprématie du fond qui ne paraît pas sur la surface qui le fait," conviction selon laquelle le fondement est la cause véritable de l'apparence, $\mathrm{d}^{\prime \prime \prime}$ argument spécieux de la métaphysique" (metaphysical fallacy). ${ }^{13}$ La

Journal of French and Francophone Philosophy | Revue de la philosophie française et de langue française Vol XX, No 2 (2012) | http://www.jffp.org | DOI 10.5195/jffp.2012.536 
philosophe de l'action veut au contraire restituer la position du common sense qui considère le monde de l'apparaître comme prioritaire.

Mis à part la pulsion de tout vivant d'apparaître, les humains se distinguent par leur capacité et leur besoin de se présenter soi-même par des actes et des mots. ${ }^{14}$ Nous nous manifestons de la manière dont nous désirons paraître et nous dissimulons ce que nous ne voulons pas qu'on voit. C'est seulement par cette auto-présentation que nous devenons pleinement individuels. Les états d'âme et leurs modifications qui caractérisent notre intériorité sont, de façon monotone, les mêmes pour tout le monde, comme il en est pour les organes internes. Cependant la littérature, à titre d'exemple, qui se rapporte à notre intériorité acquiert déjà une expression individuelle nous pouvons certes comprendre l'amour comme une sublimation de la pulsion sexuelle, de l'activité des organes internes qui demeure toujours la même, mais dans les expressions littéraires extérieures celui-ci prend des milliers de formes individuelles.

La pluralité des membres du ménage n'est pas pour Arendt la vraie pluralité des hommes égaux, affranchis des liens de nécessité du domaine familial. ${ }^{15}$ C'est uniquement dans le domaine public que la multitude de perspectives des personnes libres constitue une lumière "excédante", une clarté dans laquelle apparaît l'unicité de la personne. Alors qu'au sein du ménage nous naissons pour la première fois, nous naissons, selon la philosophe de l'action, une deuxième fois dans la lumière de l'espace public. Elle caractérise cette "seconde naissance" comme la prise de responsabilité sur notre première naissance, la naissance physique; contrairement à la naissance physique, notre initiative est ici fondamentale, bien qu'il ne faille pas négliger l'impulsion des autres.

La constitution du sujet individuel comme personne agissante libre est donc étroitement liée chez Arendt au présent de l'action: "Les hommes sont libres - d'une liberté qu'il faut distinguer du fait qu'ils possèdent le don de la liberté - aussi longtemps qu'ils agissent, ni avant ni après; en effet, être libre et agir ne font qu'un."16 Or une telle constitution de la personne qui repose purement sur le présent place Arendt face au problème suivant: comment l'identité de l'individu se conserve-t-elle en dehors des instants de l'action?

L'action se réalise au sein du monde qui sous-tend l'existence humaine par sa perpétuation (nous naissons dans un monde qui était là avant nous et qui demeurera après notre départ) et qui en même temps procure à cette existence l'intermédiaire d'une multitude d'intérêts mondains à partir desquelles l'action peut se déployer. Or, selon Arendt, l'action se crée au sein du monde sa propre sphère invisible qu'elle nomme "le réseau des relations et les histoires jouées" (das Bezugsgewebe menschlicher Angelegenheiten), ${ }^{17}$ un système de liens interhumains nés de l'action. Le réseau dispose d'une perpétuation spécifique et nous permet d'insérer notre 
action dans le réseau de modèles préétablis (qui peuvent en même temps varier en fonction de la nouveauté de l'action). Le réseau enlève à l'action sa fugacité, lui donne davantage de repérabilité et d'intégration, de sorte qu'à l'issue de notre vie d'autres personnes peuvent en retenir une histoire personnelle et la raconter. Les histoires constituent dès lors un produit involontaire de notre action, une espèce de résultante de la façon dont nous sommes apparus durant notre vie. C'est précisément ces histoires qui peuvent accéder à la perpétuation du monde des produits par leur mise en lumière: leur inscription sur des documents, leur mise en forme dans des objets usuels et des œuvres d'art. Les histoires sont également ce qui donne à notre action un sens apparent. Seule l'existence d'histoires humaines fonde également la possibilité de la grande Histoire: les Geschichten des vies individuelles créent l'espace et le modèle de la grande Geschichte de la cité elles prêtent à l'Histoire de l'humanité qui n'a à l'origine ni commencement, ni fin, ni déroulement, une qualité qui n'appartient qu'à l'action personnelle. ${ }^{18}$

\section{Michel Henry: une nouvelle détermination de l'essence du phénomène}

Pour un regard hâtif le projet phénoménologique de Michel Henry peut paraître comme un exemple-type de l'"argument spécieux de la métaphysique" dont parle Hannah Arendt, c'est-à-dire comme un cas d'élévation du fondement non-apparaissant au-dessus de l'extériorité apparaissant dans le monde commun des hommes. Cependant une recherche plus détaillée montrera que le projet de Henry, non moins critique à l'égard de la tradition philosophique que la pensée politique de la philosophe de l'action, est une autre tentative de porter un jugement positif sur la réalité effective de l'apparaître, bien que située ici dans la sphère de l'immanence, s'opposant à l'espace public.

Alors que Arendt soumet la tradition philosophique à la critique pour avoir négligé le fait de la pluralité des personnes, Henry accuse la pensée occidentale de sa tendance à penser comme vrai uniquement ce qui se montre dans la "transcendance", i.e. dans l'extériorité du monde, à l'horizon, dans le temps. Car ce type de phénoménalité suppose certaines conditions que le philosophe français englobe sous le titre de "monisme ontologique" et grâce auxquelles la philosophie occidentale semble trouver ce qui est seulement quand ceci est aliéné à soi-même. La plus marquante de ces conditions est "la distance phénoménologique" que Henry ne comprend pas ontiquement, spatialement, mais ontologico-transcendantalement comme pouvoir qui nous permet seulement de percevoir quelque chose. ${ }^{19} \mathrm{La}$ distance phénoménologique naît du travail de la transcendance qui déploie le monde comme l'horizon ultime de la visibilité: “[...] le déploiement de

Journal of French and Francophone Philosophy | Revue de la philosophie française et de langue française Vol XX, No 2 (2012) | http://www.jffp.org | DOI 10.5195/jffp.2012.536 
cette distance est un, en réalité, avec le surgissement du monde dans sa pureté." 20

Dans les conditions du monisme ontologique l'existence dépend ainsi de la distance, elle diffère de l'être. Ce qui se montre dans l'horizon du monde se montre comme distinct d'avec soi-même, le monde ne le justifie pas et ne le légitime pas, puisque les conditions de la phénoménalisation n'ont aucun lien avec le contenu du phénomène. Une chose manifestée dans le monde est "scindée d'avec soi", elle se trouve en dehors de soi et devient ainsi une simple image; privée de sa propre réalité elle apparaît comme “surface sans épaisseur, plan d'extériorité nu offert à un regard qui glisse sur lui sans pouvoir pénétrer en lui ni atteindre autre chose qu'une apparence vide." $^{21}$

La présupposition du monisme ontologique domine selon Henry la pensée philosophique occidentale dès ses commencements grecs - dans le cadre de celui-ci les choses sont définies comme des phénomènes, comme ce qui se manifeste en venant dans la lumière du monde. À l'époque moderne, certes, a surgi une nouvelle opposition entre la conscience et l'être, mais les différentes philosophies de la conscience partent en fin de compte du même présupposé de l'apparaître dans l'extériorité. ${ }^{22}$ Puisque la manifestation sous la manière de représentation est l'essence de la conscience et que la représentation présente en posant-devant, dans le milieu de l'extériorité. La subjectivité humaine prend la place de la transcendance du monde: la conscience est "le monde lui-même dans sa mondanité pure," ${ }^{23}$ son intentionnalité est l'ouverture de la distance phénoménologique et, en ce sens, la conscience transcende tout ce qui est.

La transcendance comme acte de la conscience intentionnelle de même que la transcendance comme horizon temporel dans lequel apparaissent les étants est, eu égard aux étants, néant - dans les philosophies de la conscience et dans l'ontologie heideggerienne la transcendance est le fondement grâce auquel l'étant apparaît, sans pour autant apparaître ellemême. Or Henry veut procéder de manière radicalement phénoménologique: le fondement de l'étant, l'être, s'il doit être capable d'assurer la monstration du phénomène, doit lui-même se montrer. Une construction métaphysique en elle-même non-apparaissante, seulement pensée, ne peut être le fondement, l'essence de la manifestation - le fondement de l'apparaître lui-même doit être donné positivement, doit avoir sa propre phénoménalité. C'est de cette manière que naît la conviction que l'être est identique avec l'apparaître, conviction à laquelle a également abouti Arendt, en empruntant d'autres voies.

Se manifester signifie pour Henry être accueilli, être donné à quelqu'un ou à quelque chose. La phénoménalité de la transcendance doit ainsi être assurée par l'accueil de son acte, du transcender en tant que tel. ${ }^{24} \mathrm{Il}$ s'avère toutefois que le mouvement de la transcendance ne peut pas être donné à

Journal of French and Francophone Philosophy | Revue de la philosophie française et de langue française Vol XX, No 2 (2012) | http://www.jffp.org | DOI 10.5195/jffp.2012.536 
26 L’individu comme problème phénoménologique chez Hannah Arendt et Michel Henry

cette transcendance à la façon d'une transcendance (puisqu'en elle-même elle est un se-transcender); la transcendance n'est donc pas ce qui se phénoménalise originairement dans ce mouvement. La manifestation n'est pas œuvre de transcendance, du jet de "l'essence de la manifestation" ${ }^{25}$ en dehors de soi sous forme d'horizon. En réalité, la transcendance suppose autre chose que soi (à savoir l'acte qui la constitue et qui s'accueille soimême), elle doit être accueillie pour être réelle - et un tel accueil de soi n'a lieu que dans l'immanence. "L'immanence est le mode originaire selon lequel s'accomplit la révélation de la transcendence elle-même et, comme telle, l'essence originaire de la révélation." ${ }^{26}$ Henry réserve pour cette manière originaire $\mathrm{d}^{\prime}$ apparaître dans l'immanence le concept de révélation afin d'insister sur son autonomie; la manifestation désigne désormais l'apparaître intentionnel dans la transcendance, mode d'apparaître qui dépend du mode d'apparaître originaire.

Depuis Kant nous savons que la formation d'un horizon est l'œuvre de l'imagination transcendantale, surtout temporelle, du sujet. Or, selon le philosophe français, nous ne savons rien de concret sur l'être de cette subjectivité - sont analysées les "conditions de possibilité" (de la connaissance), mais cette possibilité est vide, c'est une structure formelle privée de tout contenu. ${ }^{27}$ Le mouvement de la transcendance qui institue l'horizon est, dans le projet phénoménologique de Henry, quelque chose de dérivé, puisqu'il ne peut pas se donner à soi-même son propre contenu. L'immanence produit au contraire un contenu qu'elle accueille, elle lui est identique; le philosophe français désigne cette constitution comme l'autoaffection: accueillir un contenu signifie l'éprouver, être affecté par lui puisque le concept d" "affection" est connoté avec la prédonation passive immédiate qui caractérise la donation affective du phénomène. Cependant Henry ne veut pas penser cette donation au sens ontique d'un donné affectant qui subsiste dans l'affection, ni même au sens "ontologique" du surgissement de l'horizon du monde pur (surtout du temps pur) qui rend possible l'affection ontique et nous affecte ainsi davantage, mais dans le noyau le plus propre de l'auto-affection, dans l'épreuve absolue du soimême éprouvant: “(...) se sentir soi-même, de telle manière que le sentiment n'est pas quelque chose qui se sent lui-même, tel ou tel sentiment, tantôt celui-ci et tantôt celui-là, mais précisément le fait de se sentir soi-même considéré en lui-même dans l'effectivité de son effectuation phénoménologique, c'est-à-dire dans sa réalité." 28

L'affectivité de l'être est le fondement de l'apparaître du phénomène et l'être se manifeste dans l'immanence et non pas dans les conditions phénoménales de la transcendance du monde. Dans l'auto-affection l'affectant et l'affecté coïncident, là est la vie - l'auto-affection est une grandeur acosmique qui n'est affecté par rien d'autre qu'elle-même, par rien d'extérieur, par conséquent elle est radicalement étrangère au monde. ${ }^{29} \mathrm{La}$

Journal of French and Francophone Philosophy | Revue de la philosophie française et de langue française Vol XX, No 2 (2012) | http://www.jffp.org | DOI 10.5195/jffp.2012.536 
vie et son mode propre de phénoménalisation, l'affectivité de l'autoaffection, sont invisibles.

Henry souligne deux moments du mouvement de l'auto-affection de la vie: l'auto-donation pathique et l'ipséité. Le moment pathique se réalise dans le fait que la vie est donnée et soumise à elle-même, elle ne peut pas se défaire de 1 ' "étreinte de soi." Or par cette auto-souffrance la vie s'autoéprouve et s'auto-intensifie et jouit d'elle-même. La vie est une région ontologique dont les qualités ontologiques fondamentales sont la souffrance et la joie. La souffrance et la joie, ce faisant, ne sont pas envisagées comme des états au sens psychologique ou alors des tonalités se suivant l'une l'autre, mais comme des modes variés (ouvrant la possibilité d'une intensification de la diversité) à travers lesquels l'absolu (la vie) s'historicise, “[...] se souffrir soi-même fournissant sa matière phénoménologique au jouir de soi, se produisant comme la chair dont la Joie est faite." ${ }^{30}$ Les deux tonalités fondamentales formant l'être de la vie sont en même temps condition l'une de l'autre et l'une traverse l'autre, "la Souffrance dans la Joie et le Désespoir dans la Béatitude." ${ }^{31}$

L'autoépreuve de la vie/de l'être ${ }^{32}$ est en même temps à l'origine de l'ipséité originelle de l'apparaître et du Soi singulier de l'individu. En tant que type de subjectivité auto-éprouvante l'ipséité naît de l'identité de l'éprouvant et de l'éprouvé dans le mouvement de l'auto-affection; le Soi, toujours effectif (dans sa constitution, dans son automanifestation) et concret, "le Soi de l'essence" ${ }^{\prime 33}$ se constitue phénoménalement dans cette ipséité. L'individu est ainsi pour Henry inséparablement lié à l'autorévélation de la vie dans l'immanence et selon les divers contextes de l'exposé il reçoit différentes appellations: "l'individu", "le Soi singulier", "je peux", "Premier Vivant", "la Verbe", "le corps subjectif", "le corps-sujet", et al. Toutes ces instances - de même que la "personne" chez Hannah Arendt ont en commun l'individualité et le pouvoir d'autorévélation.

Les deux termes de la structure de la révélation sont constitutifs de la révélation et l'un est à la fois la condition originaire de l'autre. La thèse défendue dans la deuxième partie de cette étude, consacrée à M. Henry, est la suivante: l'aspect constitutif de la logique interne du projet henryen est celui qui place l'individu envisagé comme révélation du Soi concret comme l'un des termes de la structure immanente de la révélation. ${ }^{34}$ La formulation explicite de cette idée semble croître au fil de son œuvre et culminer dans sa trilogie chrétienne. La vie est donnée seulement et uniquement à travers l'ipséité. Ce n'est pourtant pas le sujet logique qui entre en scène dans l'ipséité, le sujet que l'on pense selon l'opposition sujet-objet, mais la subjectivité de l'auto-épreuve qui est inséparable du contenu de cette autoépreuve, bien qu'elle ne se confonde pas avec ce dernier et ne le "contienne" pas elle-même. 
28 L’individu comme problème phénoménologique chez Hannah Arendt et Michel Henry

\section{L'auto-génération de la vie et la seconde naissance}

La subjectivité en tant qu'ipséité de la révélation est d'après le philosophe français inscrite dans le cœur de cette dernière, dans l'immanence de la vie qui, dans son étreinte de soi, se révèle à soi-même; la structure de l'autorévélation œuvre au fondement du phénomène et nous pouvons ainsi y avoir accès dans chaque phénomène par la réduction phénoménologique. Le geste de Henry, qui fait que cette réduction devient le principe ontologique restituant toutes choses à leur unique fondement, a été précédé par celui de la littérature mystique chrétienne, dans sa tendance panenthéiste, inspirée du néoplatonisme, à chercher l'être de toutes choses dans le Dieu unique. La figure de Maître Eckhart, chez lequel la philosophe français trouve en œuvre ses propres postulats phénoménologiques, et ce dans le contexte existentiel de l'unification de l'âme avec Dieu, git au centre du chef d'œuvre qu'est L'essence de la manifestation. ${ }^{35}$

Ce passage, et bien d'autres, de L'essence de la manifestation nous mettent devant la question de savoir quel Soi Henry a-t-il en vue. Il est évident qu'il s'agit d'un Soi transcendantal, condition de possibilité de toute expérience; ce qui est moins évident c'est le statut ontologique de ce Soi - s'agit-il d'un sujet humain ou divin? Il semblerait que dans L'essence de la manifestation le statut de la subjectivité étudiée est ambigu - alternent des moments humains et divins, est étudiée l'affectivité de "notre" subjectivité (i.e. humaine), mais les conditions spécifiques de l'apparaître dans l'immanence lui donnent les qualités d'un sujet divin que sont l'éternité, l'absoluité. ${ }^{36}$ L'équivoque du statut du "Soi essence" prépare le chemin de la réinterprétation chrétienne dans l'œuvre tardive: dans C'est moi la vérité le Premier Vivant se révèle incontestablement comme la subjectivité de Dieu. Les deux autres parties de la trilogie, Incarnation et Paroles du Christ, sont consacrées au rapport entre le divin et l'humain incarnés. La réinterprétation chrétienne de la subjectivité augmente, de plus, de façon dramatique, la tension entre les deux modes fondamentaux de l'apparaître postulés dans la phénoménologie matérielle, tension présente dans le projet henryen dès le départ - l'ouverture de l'homme de l'apparaître dans l'immanence du sujet divin devient une question de vie et de mort et la clé de voûte de toute la culture humaine, une question de salut.

La métaphorique chrétienne introduit dans la phénoménologie matérielle la sphère conceptuelle de la naissance (l'engendrement, la génération, la naissance). ${ }^{37}$ L'individu naît dans l'immanence de la vie et la pluralité des Soi humains est le fruit de la vie divine: la vie se révèle à ellemême dans son auto-génération uniquement comme une ipséité individuelle; elle ne s'épuise pourtant pas en une seule étreinte de soi, mais vient à elle-même à travers d'autres étreintes de soi successives dans lesquelles naissent d'autres ipséités, de nouveaux Soi.

Journal of French and Francophone Philosophy | Revue de la philosophie française et de langue française Vol XX, No 2 (2012) | http://www.jffp.org | DOI 10.5195/jffp.2012.536 
Or l'idée d'une pluralité d'individus dans l'unité de "la vie phénoménologique absolue" pourrait impliquer la subordination des individus à la vie, la réduction des personnes individuelles à diverses expressions de la vie, etc. Il semble que c'est précisément pour éviter de soumettre les individus à une quelconque instance impersonnelle que Henry, dans son livre C'est moi la vérité, introduit sous diverses appellations un individu "premier", le "Premier Vivant", "1'Archi-Ipséité", "l'Archi-Fils", etc., qui donne la vie à elle-même dans son auto-épreuve individuelle et seulement comme telle la rend capable de donner naissance à d'autres vies et à d'autres ipséités: “[...] l'essence de l'Archi-Ipséité générée dans la Vie absolue, donnant à tout ce à quoi elle se donne de s'éprouver soi-même, elle en fait [...] un Soi absolument singulier et différent de tout autre." ${ }^{38} \mathrm{Ce}$ faisant, L'Archi-Ipséité n'est pas une "fonction" de la vie, une simple manière dont la vie viendrait à elle-même - la vie n'est pas une archi-matière ou un flux impersonnel qui se réaliserait, dans un deuxième temps, dans les individus. La vie et le (premier) individu se précèdent l'un l'autre et se donnent l'un à l'autre; dans leur coappartenance originaire, l'originarité de l'une n'empêche pas l'originarité de l'autre. Selon le philosophe français, nous ne pouvons faire valoir dans la pensée de l'immanence de la vie la représentation linéaire de la succession temporelle et causale provenant de l'imagination temporelle du "monde". ${ }^{39}$

Le caractère personnel de l'Archi-Ipséité nous oblige à ne pas la penser comme une sorte d'idée éternelle de l'ipséité, par l'intermédiaire de laquelle la vie ipséiserait tout le vivant, mais comme un événement d'autorévélation œuvrant au fondement de l'apparaître. Cet événement concret est accompagné de l'identification concrète d'une personne, dans laquelle l'inspiration néotestamentaire de l'œuvre tardive de Henry devient une identification néotestamentaire: "l'Archi-Fils", "Premier Vivant", etc., se nomme le Christ et correspond au Fils de Dieu, au Verbe incarné de l'évangile de Jean; la "vie absolue", elle, correspond à son Père. ${ }^{40}$

L'engendrement de la subjectivité humaine est inscrit dans l'engendrement de cette subjectivité divine. Henry désigne la redécouverte de la vie absolue dans sa propre vie par le concept de la théologie johannique de "seconde naissance": l'homme met de côté l'illusion égologique qu'il mène une vie autonome et s'ouvre au mouvement de la vie divine absolue dans lequel il échappe à la mort. ${ }^{41}$ L'origine de l'illusion égologique est le système de "l'égoïsme transcendantal" au sein duquel l'égo se considère comme puissance autonome de son vivre, oublie la vie et se trouve de plus en plus "dans le monde" et en se souciant de ce "monde", il se soucie en réalité de lui-même projeté dans le monde. La transformation de la seconde naissance n'est pas provoquée par une quelconque intellection théorétique, mais par le mouvement de la vie lui-même, par son autoréalisation irrévocable et prend ainsi la forme d'un faire renaissant dans lequel il n'y va plus de mes puissances, mais où il y va de l'hyperpuissance 
qui donne les actes individuels à eux-mêmes. C'est ainsi qu'il ne s'agit pas en premier lieu de ce que je fais ou pour qui je le fais, mais de l'oubli de soi et de l'entrée dans l'Archi-Ipséité de la vie absolue.

De la même manière une vraie rencontre avec autrui n'est possible qu'à travers l'auto-donation de la vie absolue en chacun d'entre nous - nous entrons chez autrui par la porte de $1^{\prime}$ Archi-Ipséité. ${ }^{42}$ L'idée de personne éthique, inacessible à ses déterminations mondaines, a pris selon Henry son origine dans le christianisme. C'est justement ici que le commandement de l'amour "Aime ton prochain comme toi-même" présent sous une certaine forme dans la plupart des cultures, trouve son fondement véritable, dans le renoncement à soi et dans l'immersion dans la personne divine qui se montre également dans le relation intersubjective et lui prête le caractère pathique propre à la vie divine.

\section{L'action dans le monde}

La conception essentiellement religieuse du sujet de Henry se reflète également dans son concept de l'action publique: l'action dans le monde, la vie politique et économique, l'éthique et d'autres formes du social sont censées, dans le projet de Henry, développer et intensifier les puissances de la vie absolue. ${ }^{43}$ La phénoménologie matérielle est du point de vue de la philosophie politique de Hannah Arendt un exemple de l'approche chrétienne qui au sein de la vita activa n'opère presque pas de distinctions et subordonne toutes les activités à un but unique, la conservation ou l'amplification de la vie dont la valeur est devenue absolue. L'anthropologie de Arendt bâtie sur son interprétation de l'expérience grecque du bios politikos est, par opposition, le déploiement de ce que Henry désigne comme la conception grecque de la "chose" en tant que phénomène se manifestant dans le monde: l'individu se dévoile aux autres dans le monde commun, dans le domaine public de l'action politique; l'individu est par essence politique. La condition de la révélation de l'agent est ainsi sa liberté d'agir.

Pour Henry, la liberté est ambivalente: elle est l'effectivité de mon “je peux", de tout ce que je peux faire, penser, etc., mais en même temps elle est passivement donnée à l'homme comme à celui qui vit dans la puissance autonome de la vie. Le lien entre l'individu et la vie est justement assuré par le religio, la religion, et c'est pour cela que toute liberté est originairement religieuse et irrévocablement, "sainte". L'égalité des hommes est dûe au lieu où l'homme est né et donc à l'auto-donation de la vie - cette auto-donation donne d'une part les individus comme inégaux, comme forts et faibles, comme hommes et femmes, plus ou moins talentueux, etc., d'autre part, elle est elle-même "l'identique" qui transforme le différent en une égalité absolue. La liberté et l'égalité ne sont donc pas des phénomènes de l'existence mondaine. Selon Henry, elles ne peuvent se réaliser que dans le

Journal of French and Francophone Philosophy | Revue de la philosophie française et de langue française Vol XX, No 2 (2012) | http://www.jffp.org | DOI 10.5195/jffp.2012.536 
lien religieux interne à la vie (i.e. dans l'éthique qui réactive le lien religieux à la vie).

\section{Conclusion}

La philosophie occidentale pense l'être en corrélation avec l'apparaître et l'objet de sa recherche est la question de savoir dans quelle mesure ce qui apparaît correspond réellement à son "fondement" causal dans l'être ou bien s'en écarte à différents degrés d'apparence, de non-vérité ou de distraction. La phénoménologie essaie de déplacer l'accent sur le pôle de l'apparaître et justifier ainsi précisément l'apparaître comme le surgissement autonome et actuel de l'étant lui-même.44 Or même sur le terrain de la recherche phénoménologique le rapport entre l'apparaître et l'apparaissant demeure en quelque sorte analogue à la hiérarchie traditionnelle de l'être et de l'apparaître: l'apparaissant est soumis aux conditions de l'apparaître qui permet et transcende les apparences individuelles mais ne se donne soimême qu'indirectement, par exemple en se cachant dans le phénomène, en ne se co-donnant que de manière incomplète, etc. ${ }^{45} \mathrm{l}^{\prime}$ apparaître (par ex. sous le titre $\mathrm{du}$ "monde") occupe dès lors une place analogue à celle qui appartenait à l'être. La pensée de Arendt et de M. Henry, chacun pour ses propres raisons, a pour but de supprimer et la distinction être-apparaître et la distinction en quelque sorte analogue apparaître-apparence: elle abroge la hiérarchie traditionnelle entre le fondement et l'apparence et place la plénitude du réel dans l'apparaître, elle considère ce qui se donne dans l'apparence comme "l'être" premier et réel qui, dès lors, ne se manifeste pas dans l'apparaître de façon imparfaite ou négative dans l'auto-dissimulation, mais, de manière présente, dans le mode unique et plein de l'autorévélation, dans laquelle le mode d'apparence se confond avec ce qui apparaît.

Bien que la structure interne de cette autorévélation requiert un schéma à au moins deux termes - la personne se révélant elle-même et la publicité chez Arendt, l'ipséité et le contenu pathique de la vie chez M. Henry - les deux auteurs ne recourent pas dans le second pôle de cette structure à une instance qui précéderait l'individu: la "publicité" de la philosophe de l'action est la pluralité des personnes, elles-mêmes agissantes et autorévélantes; "la vie" du philosophe français est le mouvement de l'autoaffection, ${ }^{46}$ dont le Soi est la porte d'entrée. L'intériorité mutuelle des deux pôles de l'apparaître permet une alternance de perspectives dans lesquelles l'un ou l'autre pôle concentre en soi tout le processus de l'apparaître - la perspective déterminante pour cette étude est celle où ce pôle est constitué par l'individu, qui porte l'apparaître en constituant son but propre.

Le concept englobant d'"individu" désigne ici deux conceptions du sujet de l'apparaître. Le concept de "personne" utilisé par Hannah Arendt souligne son rapport aux autres (dans l'action politique) que la notion de personne sous-entend dans la pensée occidentale. ${ }^{47}$ Les différents concepts employés par Michel Henry (j'en ai établi la liste plus haut) désignent au

Journal of French and Francophone Philosophy | Revue de la philosophie française et de langue française 
contraire la subjectivité du rapport à soi de la vie. Or, Henry n'hésite pas à placer au sein de cette subjectivité pathiquement constituée "une communauté de vivants", 48 une communauté invisible des Soi, qui se rencontrent sym-pathiquement dans la vie, avec laquelle ils ont la même relation transcendantale. Le philosophe français ne veut pas relier l'expérience d'autrui aux conditions de l'apparaître du monde; selon lui, autrui n'est pas donné intentionnellement dans les actes constitutifs de la conscience, mais passivement dans l'immanence de la vie absolue - la relation à autrui devient donc essentiellement religieuse. Puis, dans la trilogie chrétienne, la relation entre les Soi individuels humains est relatée comme un mouvement de retour à la source originelle de sa propre vie dans laquelle l'homme trouve également la possibilité d'une relation véritable avec les autres hommes - il forme avec eux un "corps mystique du Christ", la communauté de ceux qui sont ouverts à leur origine dans la vie divine. ${ }^{49}$

Henry et Arendt dépassent ainsi la définition traditionnelle du sujet phénoménologique comme sujet de la constitution pour lui substituer une conception événementielle. Le sujet n'est pas l'acteur de la constitution, mais il est plutôt passivement constitué (par le regard des autres qui posent, chez Arendt, la question implicite "Qui es-tu"; par l'autodonation affective du sujet et de la relation aux autres, chez Henry); et, en même temps, il est de nature dynamique, processuelle: La subjectivité affective de Henry se concrétise dans l'inversion incessante d'une tonalité phénoménologique de base en une autre, dans le passage de la souffrance à la joie; l'individualité naît chez Arendt du surplus d'apparence de l'agent et du surplus de signification de son action. En tant que passivement constitué, l'individu est, pour ainsi dire, encombré de soi-même, il est davantage que soi, et c'est dans cette surcharge qu'il se surpasse - chez Arendt vers la création de l'histoire (qui est porteuse de l'identité de l'agent dans les yeux des autres) et de là vers la possibilité de l'Histoire de la cité; chez Henry paradoxalement vers soi-même dans "l'historial de l'absolu" - dans "l'éternelle venue en soi de la vie." ${ }^{50}$

Si nous considérons la philosophie politique de Arendt comme un discours phénoménologique sui generis (j'ai essayé de convaincre le lecteur de la validité d'une telle possibilité dans la première partie de la présente étude) nous pouvons envisager sa pensée et celle de Henry comme deux tentatives majeures de défendre l'idée selon laquelle c'est l'individu (la personne/Soi) qui est le sens propre de l'apparaître, sa vérité et sa réalité la plus propre. La personne/Soi est une instance d'autorévélation qui révèle un contenu unique, individuel, hétéronome par rapport au mode d'être/apparaître de tout cosmos. Cette hétérogénéité dans le mode d'apparaître conduit les deux penseurs à quitter, dans leur recherche, le champ de "l'être" ou de la conscience intentionnelle au profit du caractère événementiel et de l'auto-donation du "phénomène" de l'individu, qui

Journal of French and Francophone Philosophy | Revue de la philosophie française et de langue française Vol XX, No 2 (2012) | http://www.jffp.org | DOI 10.5195/jffp.2012.536 
assure son apparence dans les conditions d'une subjectivité (chez Arendt le sujet de l'action politique, chez Henry la vie personnelle) qu'il instaure luimême.

Cette hétérogénéité fonde, ensuite, les projets phénoménologiques des deux auteurs comme systèmes hiérarchiques où deux sphères distinctes (deux modes) d'apparaître entrent en concurrence: le "domaine familial" (le ménage) et le "domaine public" chez la philosophe de l'action, la transcendance du "monde" et l'immanence de la "vie" chez le philosophe français. La tendance fondamentale de leurs anthropologies de justifier que l'une de ces sphères est le lieu d'une apparence pleine, maximale, à savoir de l'apparence de l'individu, alors que l'autre sphère, celle d'un mode d'apparaître affaibli ou dérivé, doit être interprétée comme une menace essentielle de l'individu. Les deux auteurs voient une telle menace dans le cours et le développement de la science et de la technique modernes. ${ }^{51}$

\section{L'individu en tant que mode intensifié de l'apparaître}

Dans l'approche de Arendt, aux niveaux inférieurs valables pour la sphère du ménage, l'apparaître part encore d'un "fondement", de l'existence biologique, de ses lois et besoins; il est apparaître d'autre chose que de soi et du contenu constitué par soi-même, et donc autovoilement, et c'est ainsi qu'il n'émet qu'une lumière faible. Or, dans la révélation de l'agent se produit un apparaître pur, sans lien avec un quelconque "fondement", constitué par l'autodonation de l'agent; l'action de l'homme dans le domaine public de la polis ne révèle pas son intériorité, c'est un fait éphémère, mais puissant, qui n'a sa raison et sa réalité qu'en soi-même. L'action politique et la parole instaurent elles-mêmes les conditions de leur possibilité et de leur accueil: du point de vue des intérêts de la vie, elle crée le surplus de lumière du domaine public des agents, de même que le surplus du sens individualisant de leurs énoncés. L'individu se révèle dans l'ex-plosion de l'action dans le surplus de lumière de la publicité et dans le sens individuel, qui sont le fruit de l'action des agents elle-même.

Dans le projet de Henry le Soi individuel est le fruit et la condition de la première autogénération de la vie, dans laquelle la vie se manifeste, dans son auto-étreinte, à elle-même (comme Premier Vivant); en même temps, cette ipséité primordiale de la vie fonde la puissance de générer d'autres vies comme des Soi auto-affectifs et se donnant à l'accusatif, qui sont "constamment auto-affectés" - le fondement de leur activité étant une passivité radicale. L'individu est donc aussi un mouvement constant de génération entre le premier vivant et d'autres vivants, qui s'ouvrent ensuite, dans la "seconde naissance", à la force divine de la première genèse. L'individu est le fruit et la condition de l'im-plosion autorévélatrice de la vie, ${ }^{52}$ invisible dans l'horizon du monde - elle agit en lui de sorte qu'il "retourne" sans cesse là où il est toujours déjà, à sa propre source.

Journal of French and Francophone Philosophy | Revue de la philosophie française et de langue française Vol XX, No 2 (2012) | http://www.jffp.org | DOI 10.5195/jffp.2012.536 
L'explosion de l'action ou l'implosion de la vie constituent des modes intensifiés de l'apparaître dans lesquelles naît l'individu en tant qu'apparence si unique qu'elle échappe à l'horizon auquel sont reliés les autres modes de l'apparaître. L'individu crée son propre milieu d'apparence et sa propre temporalité (sa propre immortalité ou éternité), paradoxale du point de vue des structures d'apparaître du "monde" et des lois de la "nature": Dans l'explosion de l'action, l'agent naît "malgré" les besoins de la vie et "sans le vouloir", dans le cadre d'une action dont l'intention ne se recoupe pas avec l'autorévélation de l'agent, dans une nouvelle qualité d'immortalité qui se manifeste uniquement aux autres dans le domaine public. L'implosion de la vie trouve la richesse inépuisable de l'individu au fond de la "pauvreté" de l'apparaître dans l'immanence, comme si le philosophe français appliquait aux condition de l'apparaître les devises de la vie monastique chrétienne: la pauvreté (se priver du monde et du systéme de "l'égoïsme transcendantal"), la chasteté (être immaculé de la transcendance) et l'obéissance (tout retourne à la souce unique de la vie absolue).

\section{L'apparaître et l'évolution culturelle moderne comme crise}

Toute hiérarchie inclut la possibilité d'un renversement. Arendt et Henry ont consacré une grande attention dans leurs œuvres au phénomène de la crise de la culture moderne, ${ }^{53}$ cette crise se produit selon les deux penseurs comme un renversement de la hiérarchie des deux modes de l'apparaître, un mode dérivé ou inférieur de l'apparaître prend la place au sommet et détermine l'auto-compréhension de l'homme, son action et le développement de la culture humaine. Les conditions phénoménales nécessaires à la naissance d'une individualité unique sont dissimulées, oubliées ou tombent en ruine. Quelque soit l'insistance avec laquelle les deux penseurs s'efforcent de distinguer le mode unique de l'apparaître de l'individu des structures impersonnelles de l'apparaître du monde (dans le cas de Henry) et de l'existence purement biologique (dans les deux cas), ${ }^{54}$ ils ne peuvent obtenir que cette distinction soit absolue.

La séparation nette entre le ménage et le domaine public dans la pratique politique grecque et romaine a selon Arendt succombé à la poussée, chrétienne et surtout moderne, de forces de certaines activités faisant partie de la vita activa - c'est précisément l'action des chercheurs accompagnée de l'œuvre de la technique qui a conduit à la perte du domaine public et de l'action en son sein, de même qu'à la perte du "monde commun" en général. Le monde commun a été remplacé par les facultés cognitives humaines (les sciences naturelles mathématisées comme connaissance fondée sur l'idéalité de la pensée) projetées dans le monde, l'action individuelle par le travail ayant pour but le bien-être social (l'accent omniprésent sur la croissance du

Journal of French and Francophone Philosophy | Revue de la philosophie française et de langue française

Vol XX, No 2 (2012) | http://www.jffp.org | DOI 10.5195/jffp.2012.536 
niveau de vie matériel), l'Histoire par les processus naturels (le but de l'œuvre technoscientifique est l'imitation des processus naturels et cosmiques). ${ }^{55}$

De manière analogue chez Henry, la même évolution moderne, dans laquelle la vie est "oubliée" et où l'homme commence à se comprendre à partir du mode dérivé de l'apparaître dans la transcendance, peut être à l'origine $\mathrm{du}$ remplacement $\mathrm{du}$ monde sensible par l'univers des particules matérielles (dans la révolution scientifique inaugurée par le travail de G. Galilée), de la "pratique de la vie" par l'action objectivée dans le Dehors du monde (l'action qui a quitté le corps et qui se produit purement dans la phénoménalité du monde comme activité des machines, des automates, des ordinateurs), du savoir pratique de la vie par le savoir théorétique de la science, de la culture par la barbarie de la technique en tant que "nature" impersonnelle et rendue indépendante. ${ }^{56}$

Or il convient ici de demander aux deux penseurs dans quelle mesure la séparation nette entre les deux sphères de l'apparaître dans leurs systèmes est tenable. L'évolution moderne n'indique-t-elle pas justement la possibilité de mêler les qualités spécifiques liées à tel ou tel mode d'apparaître? Si nous pensons avec nos philosophes la sphère de l'apparaître de l'individualité comme la sphère où l'être/la vie s'affirme de la façon la plus intense, l'évolution moderne ne se produit-elle pas comme une fécondation de la sphère du mode de l'apparaître affaibli ou dérivé par la puissance de l'autorévélation de l'individu à intensifier de façon maximale l'apparence?

Il résulte de la description de la crise moderne chez les deux auteurs que dans la région correspondante de l'être (affaibli ou dérivé) se libère une énergie jusqu'alors cachée dans le processus obscur de ses lois internes. Selon Arendt, dans l'évolution moderne il ne s'agit pas seulement de la disparition du domaine public et du monde commun, mais également de l'imitation humaine des processus naturels et cosmiques d'une telle intensité qu'elle est capable de détruire la vie de l'homme sur terre; on ne perd pas seulement la personne agissante, mais à la fois surgit une sur-famille sociale monumentale qui réclame de manière aveugle, à l'échelle étatique et aujourd'hui même mondial, son besoin biologique de croître et de consommer. $^{57}$

De la même manière selon Henry le savoir objectivé et l'action de la science et de la technique non seulement oublie la vie et opprime l'individu, le toucher, le goût, l'odeur, la couleur - et à la fois "prête" à la nature et au "monde" la puissance de la vie de se réaliser soi-même, mais privée de son caractère personnel, transformée en un processus aveugle et destructif. ${ }^{58}$ Bien que dans la sphère du mode affaibli ou dérivé de l'apparaître l'individu au sens plein de son apparence disparaît, une certaine forme de sa puissance continue à y agir et montre ainsi l'ambivalence dans le remplissement du pouvoir de cette apparence. La personne dans l'action publique ou la vie 
36 L’individu comme problème phénoménologique chez Hannah Arendt et Michel Henry

ipséisée n'éclairent et n'affirme pas uniquement soi-même ou bien d'autres qui lui sont égaux, mais potentiellement tout, y compris les régions de l'être qui dépendent originellement d'un autre régime, affaibli, de l'apparaître dans lequel les choses se donnent de manière incomplète ou progressive.

La confiance invincible des deux penseurs dans le pouvoir positif et immortel de l'apparaître doit être, selon moi, complétée par un souci accru des conditions de l'apparence qui concourent de manière substantielle à l'action pratique. Concrètement cela peut signifier deux choses: (i) l'effort de séparer au maximum les sphères des différents modes de l'apparaître. Le cas le plus visible de confusion de deux sphères de l'apparaître, et à l'échelle globale et à l'échelle locale, est l'intervention permanente des "nécessités" économiques dans la décision politique qui perd ainsi son autonomie et son pouvoir libérateur, émancipateur, individualisant. Mêler la sphère du religieux à celle du politique peut être tout aussi dangereux - l'exigence morale absolue pesant sur l'homme dans la religion peut, dans la sphère de l'action publique, éliminer la discussion et jusqu'à justifier une action violente et supprimer ainsi la condition d'égalité des hommes apparaissant dans le domaine public. Une délimitation claire de la sphère du politique et de l'auto-compréhension religieuse de l'homme pourrait être une correction apportée à la pensée de Henry par la philosophie politique de Arendt.

Par contre la sphère de l'auto-expression artistique, religieuse ou éthique de la vie devrait être indépendante des considérations politiques ou économiques qui affaiblissent la radicalité de cette expression. Le rappel du pouvoir révélant et individualisant autonome de la religion ou de l'éthique dans la phénoménologie matérielle de Henry pourrait apporter une correction à la pensée de Arendt.

On devrait enfin impartir des limites claires à la manière dont la science (et son extension, la technique) détermine l'homme. La religion, l'art, la philosophie ou bien l'action politique constituent des modes autonomes et incessibles de connaissance, de même que des manières dont l'homme crée et habite le monde. La connaissance et le pouvoir de la science ne recouvre pas toute la réalité et ne peut traduire toute la réalité, dont la réalité unique de l'individu, en l'homogénéité de son discours.

(ii) Comme l'a montré l'évolution moderne, tout en se souciant de la séparation stricte de chacune des sphères de l'apparaître, on ne peut empêcher leur mixité. Le pouvoir révélant qui accompagne la naissance de l'individu traverse toutes les frontières et peut être tout autant vivifiant que destructeur. Arendt constate une ambivalence semblable pour le domaine de l'action politique et les conséquences imprévisibles que l'action libre introduit dans le monde. ${ }^{59}$ Henry souligne au contraire la passivité de l'individu quant à son origine dans la vie divine et jusqu'à " 1 'Archipassibilité" de la vie divine elle-même. ${ }^{60} \mathrm{C}^{\prime}$ est ainsi que nous pouvons peut-

Journal of French and Francophone Philosophy | Revue de la philosophie française et de langue française Vol XX, No 2 (2012) | http://www.jffp.org | DOI 10.5195/jffp.2012.536 
être exiger pour notre action pratique un "discernement des esprits", dans lequel l'activité de notre autodétermination et de la détermination de notre monde commun tient compte de sa dépendance vis-à-vis de la donation indépassable de son fondement le plus profond et où notre activité libre, par contre, essaie sans cesse de surpasser les donations dont "l'inchangeabilité" suggère les processus dépersonnalisés des pouvoirs économiques et techniques qui veulent aujourd'hui dominer l'Homme.

1 Michel Henry a appelé ainsi son projet phénoménologique dans le dernier livre qu'il a consacré, avant sa trilogie chrétienne, à la phénoménologie théorétique. Cf. Michel Henry, Phénomenologie matérielle (Paris: Presses Universitaires de France, 1990).

2 Selon Henry: “Autant d'apparaître, autant d'être." Incarnation: Une philosophie de la chair (Paris: Seuil, 2000), 41; selon Arendt: "Etre et Paraître coïncident" La vie de l'esprit, trad. Lucienne Lotringer (Paris: Presses Universitaires de France, 1981) 37.

3 Je limite ma recherche aux trois livres suivants de Hannah Arendt: Vita activa oder Vom tätigen Leben (München: Piper \& Co., 1967), à l'origine il y avait une version anglaise, plus courte The Human Condition (Chicago: University of Chicago Press, 1958), pour la traduction française de la version anglaise Condition de l'homme moderne, trad. Georges Fradier (Paris: Calmann-Lévy, 1983); Between Past and Future: Eight Exercises in Political Thought, 1954 (New York: Penguin Books, 1968), La crise de la culture: Huit exercices de pensée politique, trad. sous la direction de Patrick Lévy (Paris: Gallimard, 1972); The Life of the Mind, I. Thinking, 1971 (je cite de l'édition de New York: A Harvest/HBJ book, 1981), La vie de l'esprit, trad. Lucienne Lotringer (Paris: Presses Universitaires de France, 1981).

${ }^{4}$ Arendt, Condition de l'homme moderne, 40.

${ }^{5}$ Arendt distingue la propriété et la richesse. La propriété est une unité spatiale (la terre, la maison), liée à la famille, qui fournit à l'homme un refuge de base. La richesse, elle, est une richesse mobile qui n'assurait pas à l'homme de l'antiquité une place stable dans le monde ni la liberté politique - même les esclaves pouvaient posséder des biens. Cf. Arendt, Condition de l'homme moderne, 70-79.

${ }^{6} \mathrm{Cf}$. Arendt, Condition de l'homme moderne, 83-85.

${ }^{7}$ Arendt, Condition de l'homme moderne, 74. 
${ }^{8}$ Arendt, La vie de l'esprit, 37.

${ }^{9}$ Arendt, Condition de l'homme moderne, 68-69.

${ }^{10}$ Lettre d'Arendt à Karl Jaspers, citée à partir de la postface de Martin Palouš, in Hannah Arendt, Krize kultury. Čtyři cvičení v politickém myšlení, trad. Martin Palouš (Praha: Mladá fronta, 1994), 155.

${ }^{11}$ Cf. Arendt, Condition de l'homme moderne, 197-204.

${ }^{12}$ Arendt, Condition de l'homme moderne, 198.

13 Arendt, La vie de l'esprit, 37.

${ }^{14}$ Cf. Arendt, La vie de l'esprit, 46-49.

${ }^{15}$ La pluralité des personnes dans le domaine public se manifeste, selon Arendt, par deux grandeurs: l'égalité et la différence. Sans l'égalité (à travers les générations, donc également envers les personnes passées et futures) il n'y aurait pas la possibilité de la compréhension, de l'action et de la parole; sans la différence entre les personnes et sans l'unicité de chacun, on n'aurait nul besoin de celles-ci. Cf. Arendt, Condition de l'homme moderne, 197-199.

${ }^{16}$ Arendt, La crise de la culture, 198.

17 Arendt, Condition de l'homme moderne, 204.

18 Arendt, Condition de l'homme moderne, 207-208.

19 Cf. Michel Henry, L'Essence de la manifestation (Paris: Presses Universitaires de France, 1963), 72-90.

${ }^{20}$ Henry, L'Essence de la manifestation, 76.

21 Henry, C'est moi la vérité: Pour une philosophie du christianisme (Paris: Seuil, 1996), 28.

22 Cf. Henry, L'Essence de la manifestation, 91-118.

23 Henry, L'Essence de la manifestation, 108.

${ }^{24}$ Cf. Henry, L'Essence de la manifestation, 206-227.

25 “L'essence de la manifestation” est, selon Henry, ce qui rend l'apparaître effectif. Elle entre en scène toujours dans le contexte ontologique et elle porte le titre d'essence parce qu'elle est autonome, indépendante de la détermination ontique. Le but de la réduction phénoménologique est, selon le philosophe français, de conduire notre recherche jusqu'à cette essence.

${ }^{26}$ Henry, L'Essence de la manifestation, 279-280.

27 Cf. Henry, L'Essence de la manifestation, 278-288.

${ }^{28}$ Henry, L'Essence de la manifestation, 578.

${ }^{29}$ Henry, C'est moi la vérité, 133-134.

Journal of French and Francophone Philosophy | Revue de la philosophie française et de langue française Vol XX, No 2 (2012) | http://www.jffp.org | DOI 10.5195/jffp.2012.536 
30

Michel Henry, La Barbarie (Paris: Grasset, 1987), 68.

${ }^{31}$ Henry, La Barbarie, 69.

32 L'essence de la manifestation, où le thème principal de la recherche était "le sens de l'être de l'ego", privilégiait la notion d'être. La trilogie chrétienne qui constitue la forme finale de la phénoménologie matérielle a quitté la recherche ontophénoménologique au profit d'une recherche purement phénoménologique. Elle privilégie la notion de "vie".

${ }^{33}$ Henry, L'Essence de la manifestation, 585.

${ }^{34}$ Ahmet Soysal a défendu une thèse similaire dans son article "Une vie en première personne," in Michel Henry, L'Epreuve de la vie. Actes du Colloque de Cerisy 1996 sous la direction d' Alain David et de Jean Greisch, (Paris: Cerf, 2001), 117-128. Paul Audi désigne l'ipséité ou l'individu transcendantal comme le point d'intérêt central de la phénoménologie de Michel Henry: Paul Audi, Michel Henry: Une trajectoire philosophique (Paris: Les Belles Lettres, 2006), 17.

35 Cf. Henry, L'Essence de la manifestation, 385-418.

36 Sébastien Laroueux a abouti à la conclusion que L'essence de la manifestation étudie la subjectivité divine: Sébastien Laroueux, L'Immanence à la limite: Recherches sur la phénoménologie de Michel Henry (Paris: Cerf, 2005), 191-197. Paul Audi, dans son livre cité plus haut, voit dans L'essence de la manifestation, de la même manière que dans Philosophie et phénoménologie du corps qui lui précède, au contraire une étude de l'individu fini, corporel et de son lien transcendantal à l'absolu.

37 Cf. Gabrielle Dufour-Kowalska, “Le corps subjectif: Incarnation et Révélation,” in Michel Henry. Pensée de la vie et culture contemporaine, éd. Jean-François Lavigne (Paris: Beauchesne, 2006), 87.

38 Henry, C'est moi la vérité, 164.

39 Cf. François-David Sebbah, "Naître à la vie, naître à soi-même. À propos de la notion de naissance chez Michel Henry," in Michel Henry, L'Epreuve de la vie. Actes du Colloque de Cerisy 1996 sous la direction d'Alain David et de Jean Greisch, (Paris: Cerf, 2001), 95-116. Mon exposé dans ces paragraphes doit beaucoup à cet article, surtout dans l'idée que “l’Archi-Ipséité” occupe une place centrale dans C'est moi la vérité justement pour qu'elle empêche la subordination des Soi à la vie.

40 Henry, C'est moi la vérité, 104-105.

41 Henry, C'est moi la vérité, 208-209 et tout le chapitre “La seconde naissance”, 192-215.

Journal of French and Francophone Philosophy | Revue de la philosophie française et de langue française Vol XX, No 2 (2012) | http://www.jffp.org | DOI 10.5195/jffp.2012.536 
42 Cf. Henry, C'est moi la vérité, 309-323.

43 Michel Henry, "Difficile démocratie," in Phénomenologie de la vie: Tome III, De l'art et du politique (Paris: Presses Universitaires de France, 2004), 167-182.

44 Cf. par ex. Eugen Fink, Sein, Wahrheit, Welt: Vor-Fragen zum Problem des Phänomen-Begriffs (Haag: Nijhoof, 1958), 131-143.

45 Cf. Renaud Barbaras, Touha a odstup: Úvod do fenomenologie vnímání (Praha: OIKOYMENH, 2005), 169-172.

46 Dans Incarnation et Paroles du Christ Henry n'utilise plus beaucoup le concept d'auto-affection et privilégie celui d' "auto-donation".

47 Prosopon en grec et persona en latin signifiait à l'origine "masque" (de théâtre), voire "visage". Cette notion a reçu une signification philosophique lors des controverses trinitaires et christologiques dans la théologie chrétienne. La distinction de chaque "personne" (gr. hypostasis ou prosopon, lat. persona) dans la Trinité divine était déterminée, dans l'œuvre des théologiens cappadociens, comme la distinction de leurs relations mutuelles - le Père est Père dans la mesure où il est en relation avec son Fils unique, etc. Cf. l'entrée "Person," in Historisches Wörterbuch der Philosophie, Band 7: P - Q, éds. Joachim Ritter, Karlfried Gründer (Basel: Schwabe, 1989), 269-338.

${ }^{48}$ Michel Henry, "Pour une phénoménologie de la communauté," in Phénomenologie matérielle, 161.

${ }^{49}$ Cf. Henry, Incarnation, 350-359.

50 Henry, "Phénomenologie hylétique et phénomenologie matérielle," in Phénomenologie matérielle, 54.

51 Cf. Arendt, La crise de la culture et Arendt, Condition de l'homme moderne, Chapitre VI. "La vita activa et l’âge moderne," 279-366; Michel Henry, Du communisme au capitalisme: théorie d'une catastrophe (Paris: Odile Jacob, 1990) et La Barbarie.

52 Pour la notion d'im-plosion de la vie (die Im-plosion des Lebens) voir Rolf Kühn et Isabelle Thireau, "Einführung in die Henrysche Kulturanalyse," in Die Barbarei: eine phänomenologische Kulturkritik, trad. Rolf Kühn et Isabelle Thireau (Freiburg/München: Alber, 1994) 30.

${ }^{53}$ Cf. note 51.

54 Le fait que Henry situe l'instance révélatrice dans la "vie" pourrait nous tenter de la comprendre de manière biologique ou biologisante: "Il faut confondre la vie phénoménologique absolue avec la vie biologique, comprendre naïvement la

Journal of French and Francophone Philosophy | Revue de la philosophie française et de langue française Vol XX, No 2 (2012) | http://www.jffp.org | DOI 10.5195/jffp.2012.536 
première à partir de la seconde, pour que l'ensemble des phénomènes de décomposition qui perturbent les organismes vivants soient transférés sur le plan des édifices sociaux, pour que le déclin et puis l'effondrement de ceux-ci apparaissent tout aussi "naturels": les civilisations sont mortelles, comme les individus, voilà tout. [...] Parce qu'ils [les individus] sont des modes de la vie absolue et portent en eux son essence, l'essence de l'auto-accroissement, la pensée de leur disparition ou de leur décrépitude n'est pas seulement vécue par eux comme un scandale: aux yeux du philosophe capable de pénétrer dans l'essence qui fait d'eux des vivants, elle se découvre comme une impossibilité d'ordre apriorique." Henry, La Barbarie, 41 (ce philosophe auquel pense Henry est Nietzsche).

${ }^{55} \mathrm{Cf}$. Arendt, Condition de l'homme moderne, Chapitre VI. "La vita activa et l'âge moderne," 279-366.

${ }^{56}$ Cf. Henry, La Barbarie, 43-100.

${ }^{57}$ Cf. Arendt, Condition de l'homme moderne, 302-308 et 360-366.

${ }^{58}$ Cf. Henry, La Barbarie, 77-99.

59 Arendt rappelle en même temps le pouvoir du pardon qui allège le poids de ces conséquences. Or elle souligne que le pardon est valable uniquement dans la sphère de l'action - par exemple, l'invasion de la technique en tant qu'œuvre industrielle dans la nature (la sphère du travail) signifie pour la nature des suites irrévocables; de la même manière, l'invasion de l'œuvre avec sa catégorie de l'utile dans l'action signifie l'invasion de moyens violents dont l'effet ne peut être réparé que de nouveau par la violence et non par le pardon (par ex. la guerre). Cf. Arendt, Condition de l'homme moderne, 265-273.

${ }^{60}$ Cf. Henry, Incarnation, 241-243.

Journal of French and Francophone Philosophy | Revue de la philosophie française et de langue française Vol XX, No 2 (2012) | http://www.jffp.org | DOI 10.5195/jffp.2012.536 